\title{
Hypertension Exacerbates Coronary Artery Disease in Transgenic Hyperlipidemic Dahl Salt-sensitive Hypertensive Rats
}

\author{
Victoria L. M. Herrera, ${ }^{1,3}$ Tamara Didishvili, ${ }^{1,3}$ Lyle V. Lopez, ${ }^{1,3}$ Kimberly Zander, $^{1,3}$ Sarah Traverse, $^{1,3}$ \\ Donald Gantz, ${ }^{2,3}$ Haya Herscovitz, ${ }^{2,3}$ and Nelson Ruiz-Opazo ${ }^{1,3}$ \\ ${ }^{1}$ Evans Department of Medicine \\ ${ }^{2}$ Department of Physiology and Biophysics \\ ${ }^{3}$ Whitaker Cardiovascular Institute, Boston University School of Medicine, Boston, MA, USA \\ Accepted October 10, 2001
}

\begin{abstract}
Background: The mechanisms underlying the known interaction of two complex polygenic traits, hypertension and hyperlipidemia, resulting in exacerbation of coronary artery disease have not been elucidated. Identification of critical pathways underlying said exacerbation could identify mechanism-based targets for intervention and prevention.

Materials and Methods: To investigate hypertensionatherosclerosis interaction, we studied the inbred transgenic atherosclerosis-polygenic hypertension Dahl salt-sensitive (S) rat model (Tg53), which over-expresses human cholesteryl ester transfer protein (hCETP) in the liver, and exhibits coronary artery disease and decreased survival compared with control non-transgenic Dahl S rats. Using serial-section histopathological and immunohistochemical analyses, we analyzed the coronary artery disease phenotype of Tg53 rats at end-stage marked by cardio-respiratory compromise as the experimental equivalent of acute coronary syndromes, and determined the effects of reduction of blood pressure through low salt diet $(0.008 \% \mathrm{NaCl})$ on the coronary artery disease phenotype and survival.

Results: End-stage $\mathrm{Tg} 53$ rats exhibit coronary artery lesions in the proximal right coronary artery system which exhibit "culprit plaque" features such as plaque inflammation, matrix degradation, apoptosis, neovascularization, thrombosis and hemorrhage recapitulating said features and heterogeneity of human coronary "culprit plaques". Comparative analysis of 6 month vs end-stage lesions
\end{abstract}

reveals distinct lesion development profiles of proximal coronary lesions which quickly progress from eccentric non-occlusive foam-cell rich lesions at 6 months to occlusive "culprit plaques", compared with more distal coronary lesions which exhibit occlusive thick-cap atheroma that remain relatively unchanged from 6 months to end stage. Reduction of hypertension through a low-salt $(0.008 \% \mathrm{NaCl})$ diet increased survival $(\mathrm{P}<0.0001)$ of $\mathrm{Tg} 53$ rats and significantly attenuated the coronary artery disease phenotype detected at 10 months of age marked by diminished apoptosis, neovascularization, matrix degradation compared with end-stage lesions detected at $<8$ months of age.

Conclusions: End stage coronary lesions in the Tg53 rats recapitulate many, albeit not all, features of "culprit plaques" in humans supporting proposed paradigms of plaque vulnerability implicating lesion macrophage enrichment, apoptosis, matrix degradation and pathological neovascularization. Comparative time course analysis of coronary lesions reveals that plaques which develop into end-stage "culprit plaques" are distinct from "stable plaques" by location and early lesion morphology, suggesting distinct lesion development and progression pathways. The significant effects of low-salt diet-induced decrease in hypertension on right coronary disease phenotype provides compelling evidence that polygenic hypertension accelerates coronary plaque progression and complication independent of cardiac hypertrophy, and more importantly provides paradigmatic support for public health policy.

\section{Introduction}

The risk of coronary heart disease (CHD) and its clinical endpoints-myocardial infarction, angina, and sudden death-or acute coronary syndromes, increases with severity of essential hypertension $(1,2)$. At any specific level of serum cholesterol between 200 and $300 \mathrm{mg} / \mathrm{dl}$, a higher incidence of CHD was observed with increasing levels of diastolic and systolic blood pressures independently (2).

Send correspondence and reprint requests to: Victoria L. M. Herrera, Whitaker Cardiovascular Institute-W-609, 700

Albany Street, Boston Massachusetts, USA 02118.

Phone: (617) 638-4020; fax: (617) 638-4066;

e-mail: vherrera@bu.edu.
Given that both essential hypertension and atherosclerosis are multifactorial polygenic diseases, the observed dose-response like effect of blood pressure on CHD is intriguingly robust. Elucidation of mechanisms underlying how hypertension exacerbates CHD is inarguably clinically relevant, as well as strategically valid to hone in on pivotal steps in the pathogenesis of acute coronary syndromes. However, elucidation has been hampered by the lack of critical animal models $(3,4)$.

Studies in established atherosclerosis models partially recapitulate hypertension-induced exacerbation of coronary heart disease as observed in humans. Hypertensive atherosclerosis models in the Watanabe hyperlipidemic rabbit exhibit increased 
aortic and coronary ostial atherosclerosis but not coronary artery disease (5). Likewise hypertensivehypercholesterolemic double eNOS/apoE knockout mice exhibit increased lesion area and plaque progression but limited to the aorta and aortic root. Although an occlusive xanthomatous lesion in the small intramyocardial artery was detected, advanced coronary artery disease and thromboses were not reported (6).

In order to gain insight into the pathogenesis of hypertension-induced exacerbation of coronary artery disease, we investigated whether the transgenichyperlipidemic/polygenic hypertension rat model expressing human cholesteryl ester transfer (hCETP), Tg53 (7), exhibits "culprit" coronary plaque features observed in human acute coronary ischemic syndromes-plaque thrombosis and hemorrhage, erosion/rupture, neutrophil adhesion, macrophage foam cell-rich, smooth muscle cell paucity, matrix degradation, neovascularization and/or apoptosis $(3,8-10)$ at end-stage defined as the overt experimental equivalent of acute coronary ischemic syndromes. In order to define the impact of polygenic hypertension on coronary artery disease, we investigated whether decreasing hypertension through a low salt $(0.008 \%$ $\mathrm{NaCl})$ diet $-\mathrm{a}$ well established finding in the transgenic model's Dahl salt-sensitive hypertensive strain background-would indeed alter the coronary lesion phenotype, and if significant, alter lifespan.

Our studies indicate that a heterogeneous spectrum of advanced plaque phenotypes recapitulating "culprit" coronary plaques in humans are detected at end-stage in $\mathrm{Tg} 53$ rat hearts, and that salt-sensitive hypertension is a major determinant of the model's robust coronary artery disease phenotype affecting multiple but interrelated pathways of inflammation, apoptosis, angiogenesis and matrix degradation.

\section{Materials and Methods}

Rat experiments were conducted according to Institutional Animal Care and Use Committee guidelines.

\section{Transgenic Rats and Experimental Manipulations}

All transgenic $\operatorname{Tg} 53$ male rats and control nontransgenic (nonTg) rats used in this study are inbred Dahl salt-sensitive (S) rats-an established genetic strain of salt-sensitive hypertension (7). The transgenic Tg53 Dahl S rat colony is maintained in our lab on regular rat chow $(0.4 \% \mathrm{NaCl})$ from weaning. Littermate negative controls are also maintained on regular rat chow. Survival of Tg53 Dahl S rats and control nonTg Dahl $S$ rats was determined on regular $(0.4 \% \mathrm{NaCl})$ rat chow. Survival of $\mathrm{Tg} 53$ rats was also determined on low salt $(0.008 \% \mathrm{NaCl})$ diet begun at 6 months of age (TD 96328, Harlan Teklad, Madison, WI). Regular rat chow and low salt diets have identical $5.4 \%$ fat, $0 \%$ cholesterol content. Survival analysis was terminated at 300 days (10 months) since survival differences reached maximum significance between $\mathrm{Tg} 53$ rats on regular rat chow compared with
$\operatorname{Tg} 53$ rats on low salt diet. No $\operatorname{lsd}-\mathrm{Tg} 53$ rat died prior to 10 months. Systolic blood pressure (SBP) was measured at 5 months of age for $\operatorname{Tg} 53$ and nonTg rats on regular rat chow prior to any cardiac compromise, and at the end of the survival analysis experiment (terminated at 10 months of age) for low salt diet fed Tg53-lsd and nonTg-lsd rats. Tail cuff blood pressure measurements were obtained by tail cuff under light anesthesia ascertaining uniform dose and duration at time of actual measurement. The average of five readings was taken.

\section{Statistical Analysis}

Survival curve analysis was done by log rank test (GraphPad Prism 3.0, San Diego, CA). Statistical analyses were done by t-test or one-way analysis of variance (ANOVA) as appropriate (GraphPad Prism 3.0, San Diego, CA) with Bonferroni's post-test for multiple comparisons.

\section{Histopathology}

Hearts were obtained after euthanasia, perfused and rinsed with ice cold PBS, and immersion fixed with aortic arch en bloc in 4\% PBS-buffered paraformaldehyde. Fixed tissues were paraffin-embedded; 6 micron serial frontal sections were obtained for end-stage Tg53 rat hearts. Approximately 200 to 300 serial frontal sections were obtained per heart for $\operatorname{Tg} 53$ endstage hearts. Hematoxylin-eosin staining was done every 10 to 15 slides. After coronary lesions were identified by microscopy, serial sections were then assigned to immunohistochemical analysis or special histological stains: Masson trichrome to distinguish smooth muscle cells (red), collagen (blue), nuclei (purple); Mallory PTAH (phosphotungstic acid hematoxylin) to detect fibrin (Prussian blue). Hearts from low salt diet fed Tg53-lsd rats were processed identically except that approximately 600 to 800 serial 4 micron cross-sections were obtained, mounted two per slide, and Masson trichrome stained every 20 slides. Diameters of arteries were measured between the two external elastic laminae on cross-section to ascertain accuracy, exclusive of the adventitia.

\section{Immunohistochemistry}

Adjacent serial sections to lesions identified on H\&E were selected and pre-determined as to order of immunostaining. Antigen retrieval and immunohistochemistry were done as described (7). Primary antibodies were obtained commercially. Antibodies used were as follows: macrophage anti-EDI (\#MAB 1435, Chemi-Con International, Inc., Temecula, CA), matrix metalloproteinase-3 or stromelysin-1 (MMP3, sc-6839), tissue inhibitor of metalloproteinases-3 (TIMP3, sc-6836), vascular endothelial growth factor (VEGF, sc-152), angiopoietin 2 (sc-7015), Caspase 3 (sc-1226) (Sta. Cruz Biotechnology, Inc., Santa Cruz, CA); active caspase 3 (G7481, Promega Corporation, Madison, WI); smooth muscle cell-specific $\alpha$-actin (A5691, Sigma-Aldrich, Inc., St. Louis, MO). Negative 
controls consisted of: a) no primary antibody on $\operatorname{Tg} 53$ rat heart sections; b) non-transgenic Dahl S rat serial rat heart sections. Specificity was determined by evident cell-specific staining and by comparative analysis of slides processed together with and without immunostaining. Negative immunostaining was validated with appropriate positive controls: cell-specific positive immunostaining in other sections treated identically. Immunostains were detected using $D A B$ chromogen (brown-black reaction product) as described (7) except for $\alpha$-actin immunostaining which was detected using red streptavidin-alkaline phosphatase substrate (Vector, Burlingame, CA). All slides were counterstained with Mayer's hematoxylin (blue nuclei).

Apoptosis was tested by in situ labeling of DNA fragmentation using terminal deoxyribonucleotide transferase (TdT)-mediated nick-end labeling (TUNEL) using the In Situ Cell Death Detection Kit (1684817, Roche Molecular Biochemicals, Indianapolis, IN) as per manufacturer's specifications using DAB (TUNEL $(+)=$ brown-black nuclei) as chromogenic detection substance. TUNEL negative (no enzyme) controls were run per manufacturer's specifications. Mayer's hematoxylin was used as counterstain (TUNEL $(-)=$ blue nuclei).

\section{Lipid Profile Analysis}

Ultracentrifugation for lipoprotein subclasses was done on fresh plasma on a potassium-bromide continuous gradient. Fractions were collected and pooled according to density $(\mathrm{g} / \mathrm{ml})$ using the following ranges validated for rat plasma in our lab: VLDL $<1.006 \mathrm{~g} / \mathrm{ml}$; IDL $1.006-1.019 \mathrm{~g} / \mathrm{ml}$; LDL $1.019-1.063 \mathrm{~g} / \mathrm{ml}$; HDL $1.063-1.210 \mathrm{~g} / \mathrm{ml}$. Cholesterol, triglyceride and apoB levels were determined using standardized kits (Wako Chemicals USA, Inc, Richmond, VA; Sigma, St. Louis, MO) as per manufacturer's specifications.

\section{Results}

\section{End-stage Tg53 Coronary Lesions Recapitulate Human "Culprit" Coronary Plaques}

We investigated male Tg53 rats maintained on regular rat chow (designated as $\operatorname{Tg} 53$ throughout manuscript) at end-stage defined by the onset of spontaneous cardio-respiratory distress prompting euthanasia. This is taken as the experimental equivalent of acute coronary ischemic syndromes in humans. In order to eliminate confounding factors, hearts from Tg53 rats that exhibited cerebro-vascular disease or that died prior to euthanasia were excluded from histopathological analysis. $\operatorname{Tg} 53$ rats were housed in pathogen-free conditions in order to eliminate infection as a confounder. Analysis of end-stage $\operatorname{Tg} 53$ rats revealed severe coronary artery disease in the proximal coronary artery in all seven rats analyzed associated with varying ages of right ventricular myocardial infarction. Lesion analysis is limited to the right coronary artery system. Coronary lesions are more advanced in the right coronary artery system compared with the left coronary artery system.

As observed in human acute coronary syndromes, we detected lesion heterogeneity with at least four lesion phenotypes in end-stage $\mathrm{Tg} 53$ rat hearts. Representative composites of heart serial sections from end-stage Tg53 male rats reveals similarities with "culprit" lesions observed in human acute coronary syndromes. Figure 1 shows two Tg53 coronary atherosclerotic lesion phenotypes surrounded by infarcted myocardium exhibiting fibrosis, myocardiocyte loss and inflammation. Lesion thrombosis is detected in several serial sections (Figs. 1A-D) corroborated by phosphotungstic acid hematoxylin (PTAH)-stained fibrin (Fig. 1C). A non-thrombosed, occlusive $(>75 \%)$ plaque (\#2 in Figs. 1A, E-H) exhibits features of "culprit"-prone or "vulnerable" plaques observed in human acute coronary syndromes $(8,10)$ such as: lipid-rich core, thin fibrous cap with paucity of smooth muscle cells and collagen (Fig. 1E), induced expression of matrix degrading metalloproteinase (MMP) expression $(8,11)$ with deinduced expression of tissue inhibitors of matrix metalloproteinases (TIMP) (8), as seen in the intense stromelysin (MMP3) expression (Fig. 1G) and minimal staining for tissue inhibitor for matrix metalloproteinase-3, TIMP3 (Fig. 1H). Specificity of MMP3 immunostaining is demonstrated by differential and site specific staining in three different serial sections (Figs. ID, G, I), as well as reproducibility in other complication-prone lesions (data not shown). Specificity for PTAH is ascertained by the detection of fibrin in Fig. IC but not in the nonthrombosed "culprit-prone" plaque (Fig. IF).

A third lesion phenotype observed (Fig. 2) also exhibits multiple features of "culprit- or culpritprone" plaques associated with acute coronary syndromes in humans $(3,8,10)$. Intraplaque thrombi are detected with largest plaque thrombus at the proximal lesion shoulder (Fig. 2A-D) corroborated by PTAH-stained fibrin (Fig. 2C). The lesion exhibits abundant foam cells, extracellular lipid (Fig. 2A-D, F-I) and intraplaque hemorrhage (Figs. 2B, F). Overlying the thrombosed shoulder region, the plaque cap is thin with sparse smooth muscle cells (Fig. 2D), in contrast to the distal coronary lesion (same rat heart section) with thick cap and abundant $\alpha$-actin stained smooth muscle cells (Fig. 2E) simulating "stable" plaques (10). High power magnification reveals active inflammation with multilobulated neutrophil adhesion onto activated plaque endothelium (Figs. 2B, F, G, I) in the proximal (Fig. 2B, F) and distal (Fig. 2G) shoulders of the lesion, as well as adjacent to an area of rupture detected in adjacent serial sections (Fig. 2H-I). This advanced complex lesion is EDl+ macrophage-foam cell enriched (Fig. 2H). MMP3 expression is detected in both smooth muscle cells and macrophages (Fig. 2I). MMP3 + macrophage-foam cells underlie the rupture 

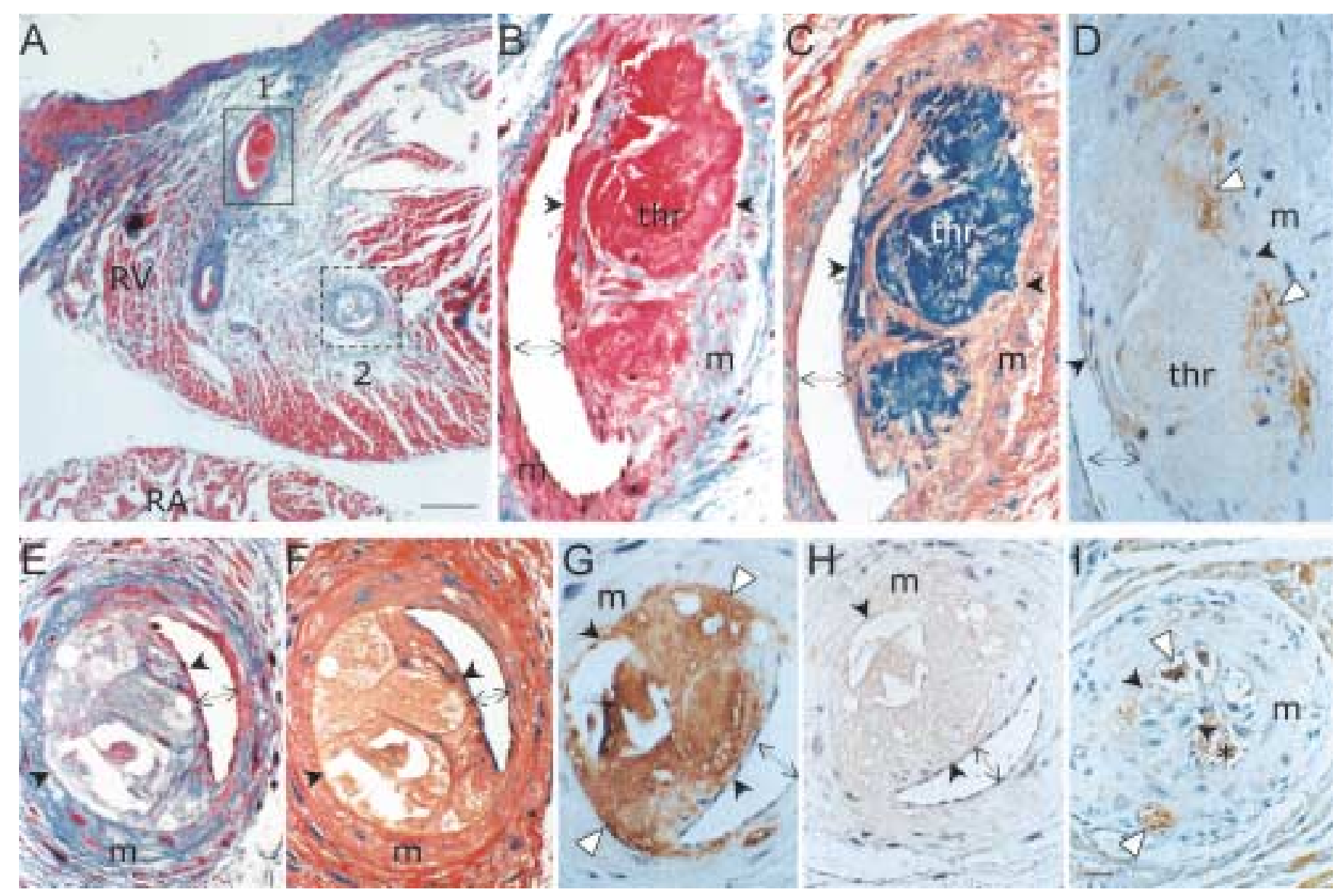

Fig. 1. Composite of two end-stage Tg53 proximal coronary ( $\approx 200$ micron) lesion phenotypes. (A) Low magnification Masson trichrome-stained section reveals an occlusive, thrombosed lesion (1) and an occlusive complication-prone non-thrombosed lesion (2) in the right ventricle (RV). Boxed lesion $1(B-D)$ and lesion $2(E-H)$ are further analyzed in adjacent serial sections; RA, right atrium; bar $=200$ microns. (B) High magnification of occlusive plaque 1 reveals endothelialized thrombus and few smooth muscle cells in the lesion-side media, in contrast to abundant smooth muscle cells in the non-lesion-side media. (C) PTAH stained fibrin in thrombosed plaque. (D) MMP3 immunostaining at the base $(\triangleright)$ of thrombosed plaque. (E) High-magnification of lesion 2 shows few smooth muscle cells in the plaque and lesion-side media, acellular plaque core with large extracellular lipid deposits; significant amount of collagen (dark blue) in the lesion-side media; minimal collagen in plaque core (pale blue) and in non-lesion side media. (F) No PTAH-stained fibrin in plaque 2. (G) Marked MMP3 immunostaining in plaque 2 core $(\triangleright)$; few stained cells in media.

(H) Negative TIMP3 immunostaining in plaque 2 corroborates specificity of MMP3 immunostaining (D, G). (I) Occlusive distal coronary $(\approx 160$ microns) lesion with minimal MMP3 immunostaining (identical to $(D, G)$ ) restricted to macrophage-foam cells $(\triangleright)$ in the lesion and media; $*$, non-specific staining of red blood cells; bar $=20$ microns. Note: $(\leftrightarrow)$, residual lumen; $(><)$, plaque; (m), media; (thr) thrombosed plaque.

site supporting the inflammation-proteolysis hypothesis in plaque rupture $(8,10)$. In addition to rupture, endothelial erosion is also detected in the lesion shoulder with overlying leukocyte adhesion and subjacent intraplaque microthrombi (Fig. 2G).

A fourth lesion phenotype observed (Fig. 3) exhibits smc-rich thick fibrous cap (Fig. 3A, B) consistent with "stable plaque" features (11) but with intraplaque hemorrhage (Fig. 3A, B) and thrombosis (Fig. 3C) in areas of EDl-immunostained macrophage foam cells (data not shown). Lesion neovascularization was investigated to account for plaque hemorrhage and thromboses. Immunohistochemical analysis detects vascular endothelial growth factor (VEGF) (Fig. 3D) and angiopoietin-2 (Fig. 3E) expression in EDI+ (data not shown) macrophage foam cells in areas of plaque neovascularization, hemorrhage and thrombosis. On high magnification, ectatic thin-walled neovessels are detected in association with confirmatory red blood cells intraluminally (Figs. 3F-H). Neovessel endothelium stains with VEGF (Figs. 3F- G) but not with angiopoietin-2 (Fig. 3H). Neo-vessels are located in the intimal lesion (Figs. 3E-H), as well as traversing from the media to the intima through areas of disrupted internal elastic laminae (Fig. 3D, F). The expression of both VEGF and angiopoietin-2 implies immature unstable vessels (12) consistent with the hypothesis that lesion neovascularization not only leads to influx of inflammatory cells (13) but also leads to intraplaque hemorrhage and thrombosis resulting in plaque destabilization. VEGF expression has been detected and implicated in human coronary 


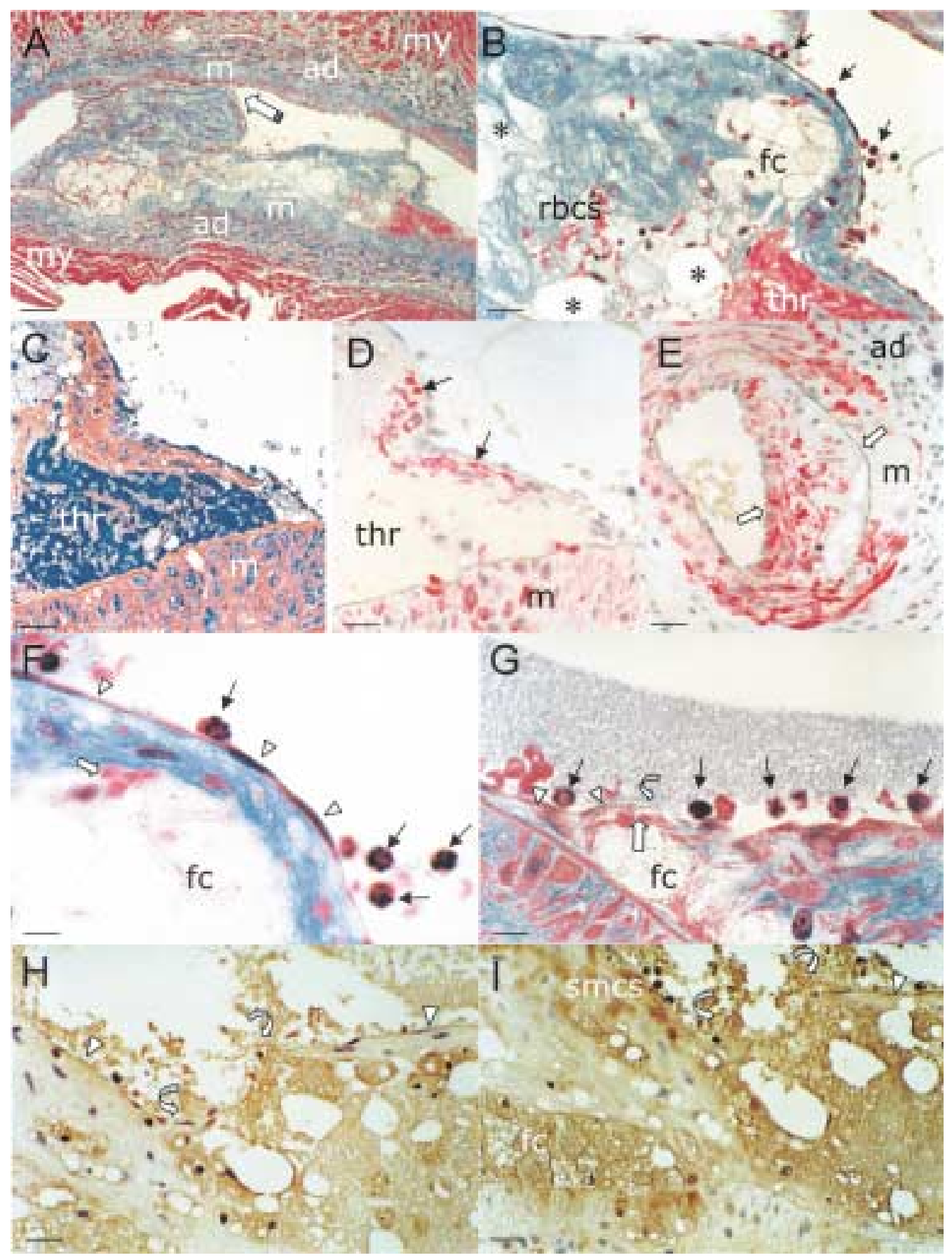

Fig. 2. Composite of another end-stage Tg53 proximal coronary ( $\approx 320$ microns) lesion phenotype: foam-cell rich, plaque rupture, erosion, hemorrhage and thrombi. (A) Masson-trichrome stained section reveals longitudinal profile of occlusive foamcell enriched plaque with intraplaque hemorrhage and thrombosis most prominent in the proximal plaque shoulder; direction of blood flow (仓仓); bar $=200$ microns. (B) High magnification of lesion shoulder reveals thrombus, foam cells, globular extracellular lipid deposits $(*)$, red blood cells, and multilobular neutrophil $(\rightarrow)$ adhesion on the endothelium. Bar $=20$ microns. $($ C) PTAH stained fibrin in the lesion shoulder thrombus; bar $=20$ microns. (D) Few $\alpha$-actin immunostained smooth muscle cells $(\rightarrow)$ overlying thrombosed lesion shoulder; (bar $=20$ microns). (E) In contrast, fibroproliferative lesion $(\Leftrightarrow \Leftarrow)$ in a distal coronary artery (identical section to (D)) reveals abundant smooth muscle cells (red) in the lesion cap and media; bar $=20$ microns. (F) High magnification (bar $=8$ microns) shows multilobular neutrophil $(\rightarrow)$ adhesion onto intact endothelium $(\nabla)$; red blood cells $(C)$ in plaque; foam cells close to lesion surface. (G) High magnification (bar $=8$ microns) reveals endothelial erosion $(\hookrightarrow)$ in the distal lesion shoulder with adhered neutrophils and lymphocytes $(\rightarrow)$, and subendothelial thrombi $(\diamond)$ which stained for fibrin (PTAH data not shown). (H) ED l immunostained macrophage-foam cells are detected close to site of rupture ( $\subset$ ) and throughout lesion. Large extracellular lipid globules (delipidated during processing) are noted; bar $=20$ microns. (I) Adjacent serial section reveals MMP3 immunostained smooth muscle cells, macrophage foam cells, and rupture site $(\zeta)$; bar $=20$ microns. Note: $(\nabla)$, lesion surface with intact endothelium; (ad), adventitia; (fc), foam cells; (m), media; (my), myocardium, (rbcs), red blood cells; (smcs), smooth muscle cells; (thr), thrombus. 


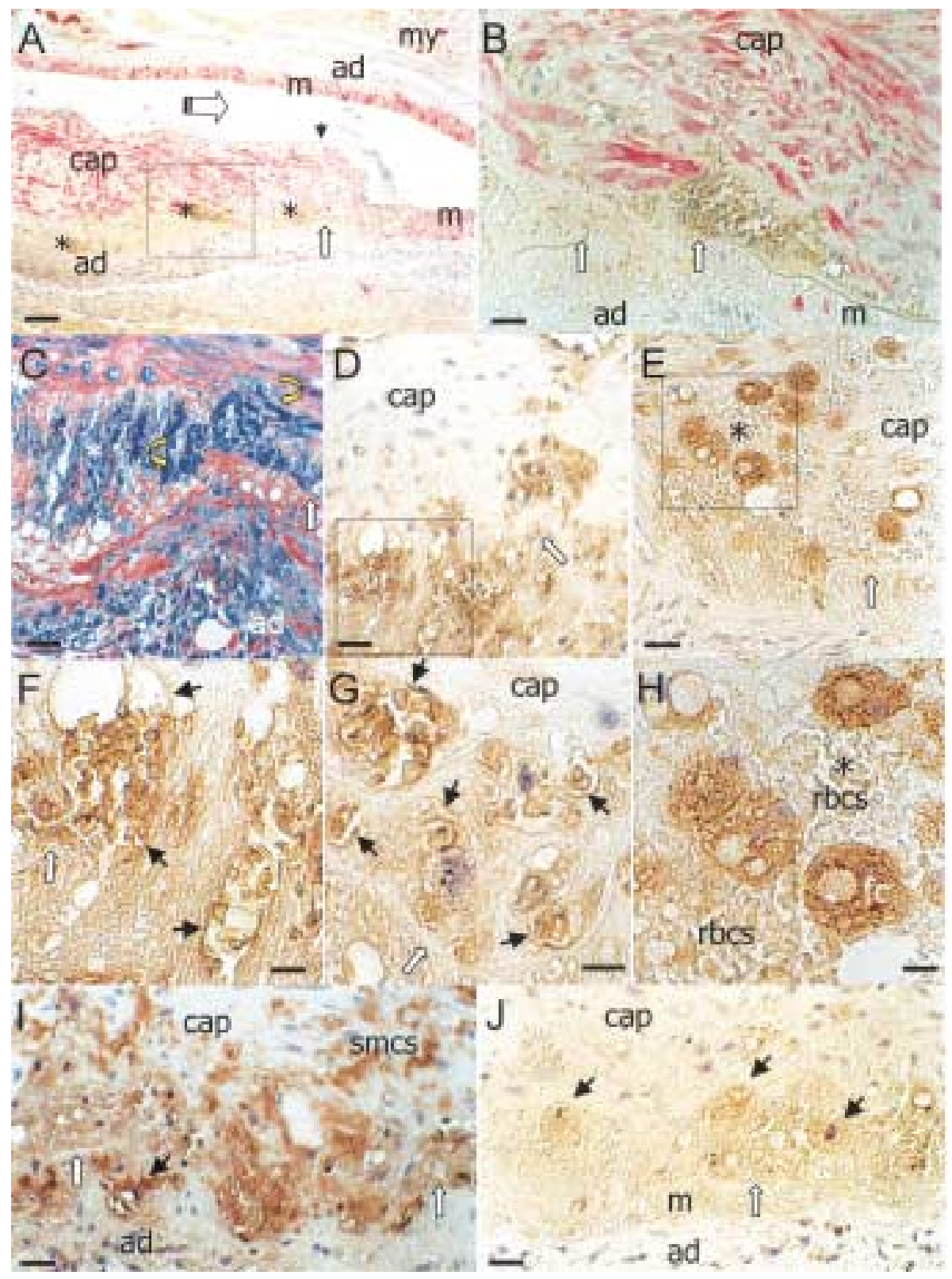

Fig. 3. Composite of a fourth Tg53 proximal coronary ( $\approx 350$ micron) lesion phenotype found at end-stage: thick smooth muscle cell-rich cap, intraplaque hemorrhage, thrombosis, disrupted internal elastic laminae. (A) Abundant $\alpha$-actin immunostained smooth muscle cells (red) in plaque cap and media of opposing non-lesion side, in contrast to paucity in lesion-side media. Direction of blood flow ( $)$ ); endothelium $(\rightarrow)$; internal elastic lamina $(\Leftrightarrow)$. Areas of hemorrhage are detected at the plaque base (boxed) and in subjacent media; bar $=100$ microns. (B) High magnification of boxed area in (A) reveals numerous $\alpha$-actin immunostained smooth muscle cells in the plaque cap; disrupted internal elastic lamina $(C)$ and intraplaque hemorrhage (red blood cells stained green-brown). Minimal $\alpha$-actin immunostained smooth muscle cells in the lesion-side media; bar $=25$ microns. $($ C) PTAH + fibrin $(\hookrightarrow)$ is detected in areas of plaque hemorrhage $(*)$; bar $=25$ microns; disrupted internal elastic lamina $(\Longrightarrow)$; delipidated extracellular lipid globules are noted. (D) VEGF immunostained macrophage foam cells and endothelial cells; none in lesion cap smooth muscle cells. Neovessels filled with red blood cells surround VEGF $(+)$ macrophage foam cells; disrupted internal elastic lamina $(\Longrightarrow)$; bar = 25 microns; high magnification of boxed area in (F). (E) Angiopoietin-2 immunostained macrophage foam cells are surrounded by neovessels filled with confirmatory red blood cells $(*)$; disrupted internal elastic lamina $(\Longrightarrow)$; high magnification of boxed area in $(\mathrm{H})$; bar $=25$ microns. (F and G) High magnification of VEGF immunostained ectatic neovessel endothelium $(\rightarrow)$ with confirmatory intraluminal red blood cells; internal elastic lamina $(\Rightarrow)$; bar $=10$ microns. $(\mathbf{H})$ High magnification of angiopoietin-2 immunostained macrophage foam cells with surrounding red blood cell pools $(*)$; bar $=10$ microns. (I) MMP-3 immunostained smooth muscle cells (smcs) and macrophage foam cells $(\rightarrow)$ in the plaque and media; minimal immunostaining in adventitia; disrupted internal elastic lamina $(\Longrightarrow)$. (J) Minimal TIMP3 immunostaining confined to macrophage foam cells $(\rightarrow)$; internal elastic lamina $(\Longrightarrow)$; bar $=20$ microns. Note: (ad), adventitia; (cap), plaque cap; (fc), foam cells; (m), media; (my), myocardium; (rbcs), red blood cells; (smcs), smooth muscle cells. 
plaque progression (14). Significant MMP3 expression in both macrophage foam cells and intimal smooth muscle cells (Fig. 3I) with minimal TIMP3 expression (Fig. 3J) suggests a pathological imbalance towards matrix degradation with MMP3-to-TIMP3 ratio greater than one.

\section{Prelude to the "Culprit" Plaque}

A key issue in human coronary artery disease is the determination of the pre-"culprit" plaque phenotype. The analysis of genetically identical transgenic rats under controlled identical experimental conditions at different time points provides a valid experimental paradigm to investigate a continuum of lesion development. Comparison of end-stage lesions with lesions observed at 6 months reveals that the "culprit" lesions in end-stage $\operatorname{Tg} 53$ rats are in the identical location of 6-month proximal coronary lesions exhibiting non-occlusive eccentric lipid-rich lesions with intimal foam cells and prominent leukocyte adhesion (Fig. 4A) that are EDl+ (Fig. 4B). These early lesions have no intimal smooth muscle cells (Fig. 4C). In contrast, the more occlusive fibroproliferative lesion in the more distal right coronary artery (Fig. 4D), remained either the same or slightly more occlusive but did not exhibit any plaque complication features such as erosion, hemorrhage or thrombosis (Fig. II and 2E). This deter- mines the prelude lesion phenotype of the "culprit" end-stage lesion in Tg53 rat model as eccentric, nonocclusive, macrophage-foam cell rich lesion with prominent endothelial leukocyte adhesion and adventitial inflammatory cells. At 6 months, this preculprit lesion seems far less advanced than the fibroproliferative lesion detected quite reproducibly in the more distal coronary artery.

\section{Impact of Hypertension on Tg53 Rat Survival}

In order to dissect the role of hypertension in coronary lesion progression in Tg53 Dahl S rats, we placed 6-month old Tg53 male rats on a low salt diet, Tg53-lsd. When Dahl S rats are placed on a low salt diet $(0.008 \% \mathrm{NaCl})$, blood pressure is lowered (15) and life expectancy normalized. Lesion initiation is not addressed here since at 6 months, lesions are already present (7). As shown in Fig. 5, survival curve analysis detects increased survival of $\mathrm{Tg} 53$-lsd rats compared with $\mathrm{Tg} 53$ rats on regular rat chow (Tg53reg). Analysis of systolic blood pressure (SBP) by tail cuff revealed that after 4 months on a low salt diet, 10m-old Tg53-lsd rats have reduced hypertension similar to age-matched nonTg-lsd rats (Tg53-lsd $\mathrm{SBP}=157 \pm 9$ s.e.m., $\mathrm{n}=7$; nonTg-lsd SBP $=148 \pm$ $4 \mathrm{mmHg}, \mathrm{n}=6 ; \mathrm{P}>0.05)$. SBP on low salt diet is significantly reduced (multiple comparisons $\mathrm{P}<0.001$ ) compared with SBP levels in both Tg53-reg rats and

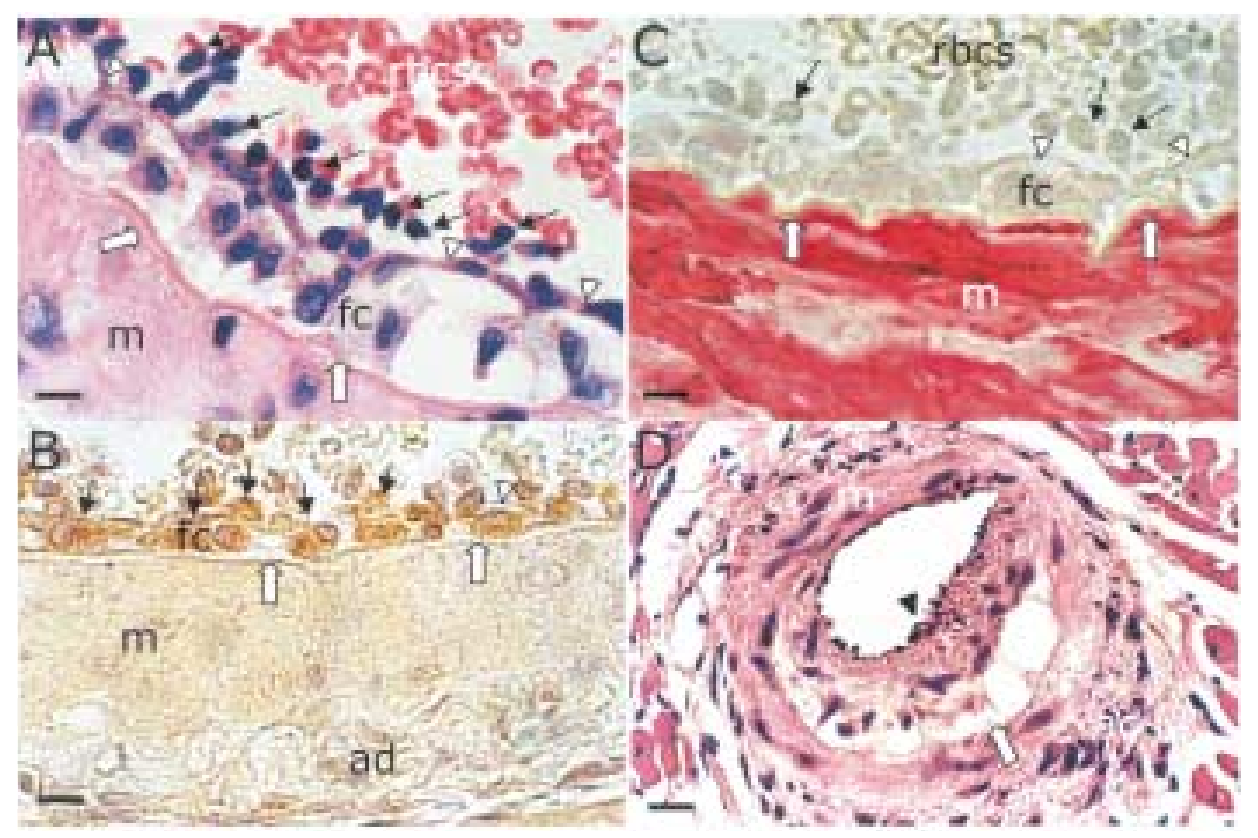

Fig. 4. Representative composite of 6 month-old non-end-stage Tg53 proximal ( $\approx 400$ micron) coronary lesion. (A) H\&E staining reveals foam cell $(\mathrm{fc})$-rich lesion; intact internal elastic lamina $(\Leftrightarrow)$; leukocyte adhesion $(\rightarrow)$; intact endothelium $(\nabla)$; red blood cells (rbcs) in the vessel lumen; media (m); bar $=10$ microns. (B) EDl immunostained $(\rightarrow)$ lesion foam cells and adhered leukocytes indicating monocyte-macrophage lineage; intact endothelium $(\nabla)$; internal elastic lamina $(\vec{C})$; bar $=10$ microns. $(\mathbf{C})$ Intense $\alpha$-actin immunostained (red) smooth muscle cells in the lesion-side media; no immunostaining in intimal lesion; leukocyte adhesion ( $\rightarrow$ ) onto the intact endothelium $(\nabla)$; bar $=10$ microns. (D) HEE stained distal coronary ( $\approx 160$ microns) plaque with thick cap, delipidated extracellular lipid, intact endothelium $(\nabla)$ and internal elastic lamina $(\vec{b})$. No thrombi, hemorrhage or neovascularization are detected. Identical H\&E stained section to (A); bar = 25 microns. Note: (ad), adventitia; (fc), foam cells; (m), media; (rbcs), red blood cells. 


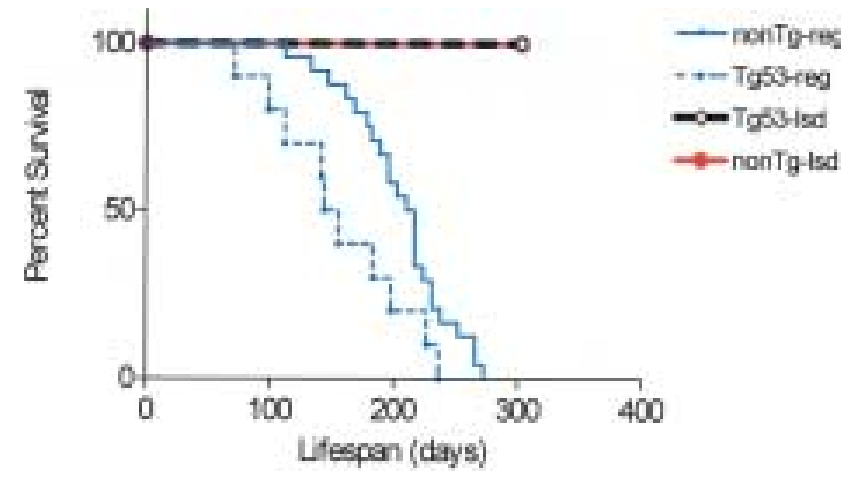

Fig. 5. Survival curve analysis of Tg53 rats on regular salt $(0.4 \% \mathrm{NaCl})$ diets, Tg53-reg rats, compared with $\mathrm{Tg} 53$ rats on low salt $(0.008 \% \mathrm{NaCl})$ diets from 6 months of age, Tg53-Isd rats. Lifespan is plotted in days $v s$. percent survival up to 300 days. Tg53-lsd rats exhibit increased survival compared with Tg53-reg rats $(\log$ rank test $\mathrm{P}<0.0001)$. Since maximum probability was reached at 300 days, the Tg53-lsd rat survival experiment was terminated and hearts analyzed at this time point. Survival is decreased $(\log$ rank test $P=0.0004)$ in Tg53 rats (Tg53-reg) compared with nonTg Dahl S rats (nonTgreg) on regular rat chow, corroborating earlier observations. ${ }^{7}$ Lifespan of low salt diet fed Tg53-lsd and nonTg-lsd rats are increased (multiple comparison $P<0.001$ ) compared with regular rat chow fed Tg53-reg and nonTg-reg rats respectively.

nonTg-reg rats at 4 months (data not shown) and at 5 months (Tg53: $\mathrm{SBP}=196 \pm 5$ s.e.m., $\mathrm{n}=12$; nonTg: SBP $=196 \pm 4 \mathrm{mmHg}, \mathrm{n}=5$ ). Lipid analysis revealed equivalent severe combined hyperlipidemia, as well as similar distribution of cholesterol and triglyceride in different lipoprotein classes in $\mathrm{Tg} 53$-lsd rats and $\mathrm{Tg} 53$-reg rats (Table 1).

Normolipidemic, hypertensive nonTg-reg rats have longer lifespan than hyperlipidemic, hypertensive Tg53-reg rats, but have shorter lifespan compared with low-salt diet fed Tg53-lsd rats which remain hyperlipidemic but with reduced hypertension (Fig. 5). This observation is not surprising given that untreated hypertension in humans, similar to untreated hypertension in end-stage nonTgreg rats, can lead to cardiac mortality prior to or without coronary lesions. As expected, reduced hypertension in nonTg-lsd rats results in increased survival compared with nonTg-reg rats (Fig. 5). Both Tg53-lsd and nonTg-lsd rats are well at 10 months. Experiments were then terminated to assess lesions at a time point with survival differences already at maximum significance. Since Dahl $S$ rats on low salt diets from weaning are normotensive and expected to have normal life spans (15), the comparative survival analysis between Tg53-lsd and nonTg-lsd Dahl S rats requires separate study.

\section{Impact of Hypertension on Tg53 Coronary Lesion Phenotype}

In order to investigate the impact of polygenic hypertension on coronary lesion development, we compared proximal coronary lesions from symptomatic end-stage Tg53 rat hearts and asymptomatic nonend-stage Tg53-lsd rat hearts (obtained at 10 months). Analysis revealed lesion heterogeneity in both, but with an overall decrease in lesion complexity and lumen occlusion in Tg53-lsd proximal coronary lesions compared with end-stage Tg53 rats. Three representative lesion phenotypes of $\mathrm{Tg} 53$-lsd rats are shown in Fig. 6.

One lesion phenotype (Fig. 6A-D) exhibits intimal foam cell-rich lesion with prominent leukocyte adhesion and adventitial inflammatory cells (Fig. 6A, C), no intimal $\alpha$-actin stained smcs (Fig. 6B). This lesion phenotype resembles the 6 month-Tg53 proximal coronary lesion phenotype (Fig. 4), but differs in that the 10 month-Tg53-lsd lesion has

Table 1. Lipid profile of control and Tg53 rats on regular $(0.4 \% \mathrm{NaCl})$ and low salt $(0.008 \% \mathrm{NaCl})$ diets

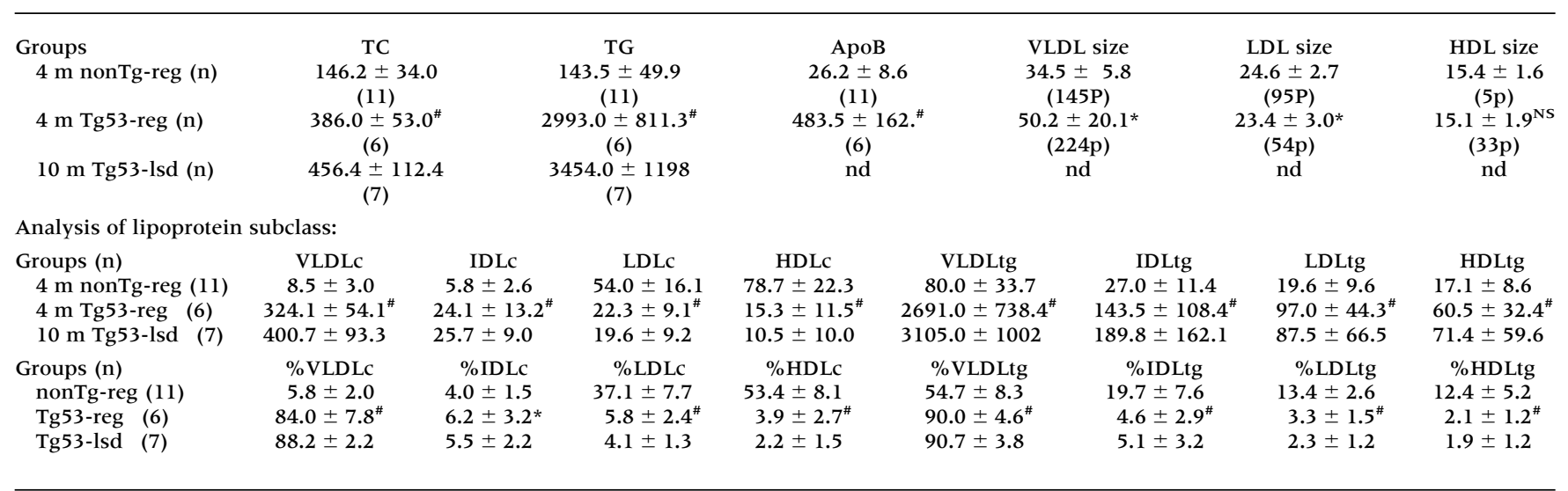

\#, ANOVA P $<0.0001 ;$ *, t-test $\mathrm{P}<0.05$; NS, not significant; lipid profile presented as mean \pm s.d. mg/dl; nd, not done; non-Tg, non-transgenic Dahl S rat controls on regular $(0.4 \% \mathrm{NaCl})$ rat chow; $\mathrm{Tg} 53$-reg, $\mathrm{Tg} 53$ rats on regular rat chow; Tg53-lsd, $\mathrm{Tg} 53$ rats on low salt $(0.008 \% \mathrm{NaCl})$ diet. ApoB, apolipoprotein $\mathrm{B}$; c, cholesterol in subclass; $\mathrm{HDL}$, high density lipoprotein; IDL, intermediate density lipoprotein; LDL, low density lipoprotein; n, number of rats or particles (p); TC, total plasma cholesterol; TG, total plasma triglyceride; tg, triglyceride in subclass; VLDL, very low density lipoprotein. 


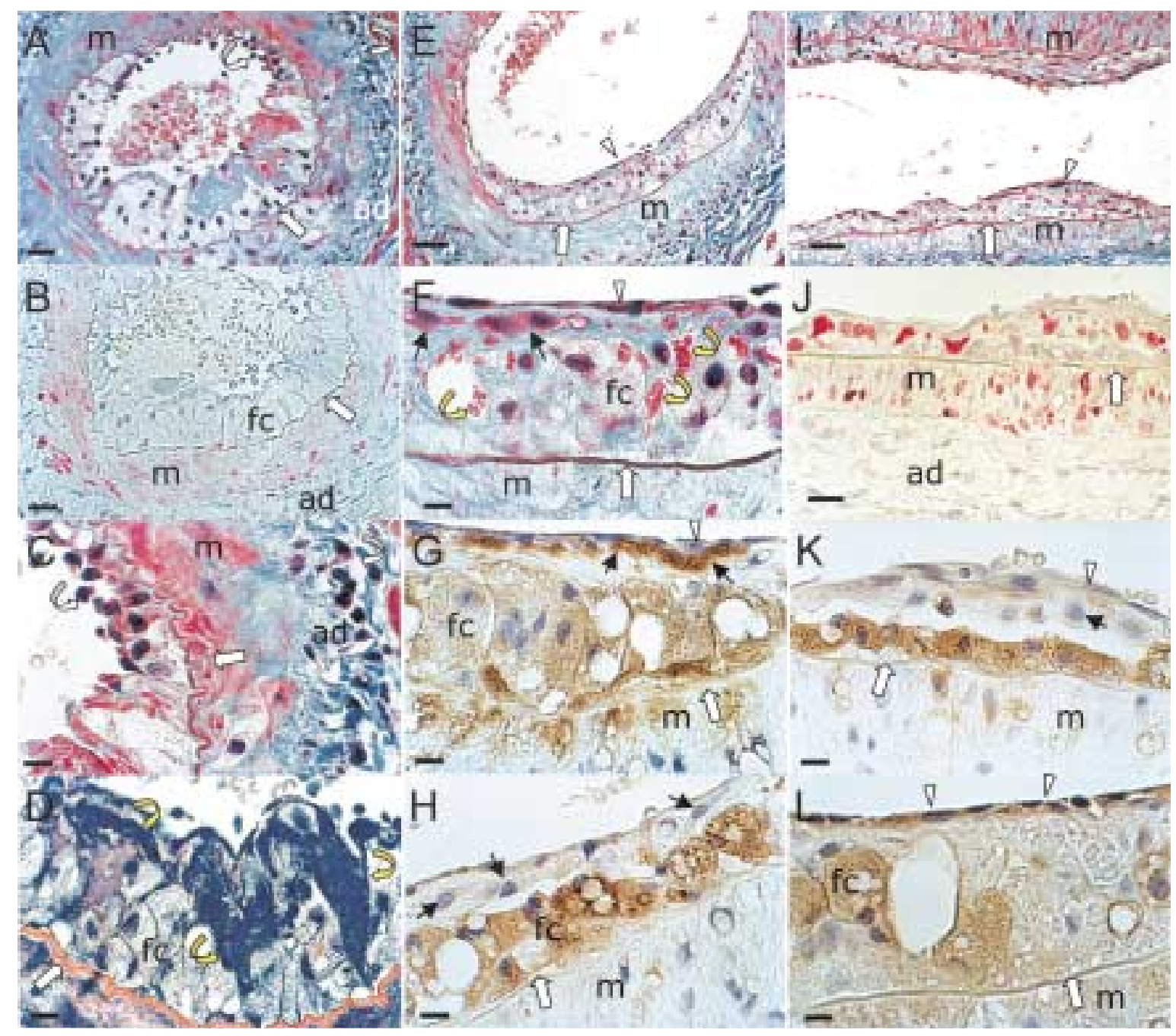

Fig. 6. Three representative proximal coronary lesion phenotypes detected in 10 month-old Tg53-lsd rats. (A) One type of Tg53-lsd proximal coronary ( $\approx 200$ microns) lesion comprised predominantly of macrophage foam cells with collagen-rich areas (blue), inflammatory cell ( $\hookrightarrow$ ) adhesion at the shoulder and presence in adventitia (ad); internal elastic lamina is intact; bar = 25 microns; Masson trichrome stain. (B) $\alpha$-actin immunostained (red) smooth muscle cells in the media; none in lesion. Internal elastic lamina is intact; lesion foam cells are macrophage $\mathrm{EDI}(+)$ (data not shown); bar $=25$ microns. (C) High magnification reveals intact internal elastic lamina; inflammatory cell $(C)$ adhesion at lesion shoulder and presence in adventitia; bar $=10$ microns. (D) PTAH-stained fibrin in intraplaque thrombi (yellow $\hookrightarrow$ ); internal elastic lamina is intact; bar $=10$ microns. (E) A second proximal coronary $(\approx 350$ microns) lesion phenotype: eccentric intimal lesion with smooth muscle cells. Few smcs are noted in lesion-side media compared to non-lesion side media; bar $=50$ microns; Masson trichrome stain. (F) High magnification of E reveals intact endothelium and internal elastic lamina, foam cells, intimal smooth muscle cells $(\rightarrow$ ), intraplaque red blood cells and microthrombi (yellow $\hookrightarrow$ ); bar $=10$ microns. (G) MMP3 immunostaining in lesion smooth muscle cells $(\rightarrow)$ and macrophage foam cells; light MMP3 immunostaining detected in lesion-side media; endothelium is intact; bar $=10$ microns. (H) TIMP3 immunostaining in macrophage foam cells; none in lesion smooth muscle cells $(\rightarrow)$; bar $=10$ microns. (I) A third proximal coronary $(\approx 350$ microns) lesion phenotype that is minimally occlusive; bar $=50$ microns. $(\mathrm{J}) \alpha$-actin immunostained smooth muscle cells (red) in intimal lesion and lesion-side media; bar $=25$ microns. (K) MMP3 immunostaining in macrophage foam cells but not in intimal smooth muscle cells $(\rightarrow)$; some staining in media smooth muscle cells and foam cells; bar $=10$ microns. (L) VEGF immunostaining in macrophage foam cells and endothelial cells $(\nabla)$ in lesion analyzed in panel E; no VEGF+ neovessels; delipidated extracellular lipid; bar $=10$ microns. Note: Endothelium $(\nabla)$; internal elastic laminae $(\Longrightarrow)$; $(\mathrm{ad})$, adventitia; (fc), foam cells; (m), media.

increased in size and has plaque thrombi confirmed on PTAH fibrin-staining (Fig. 6D). A second lesion phenotype (Fig. 6E-H \& 6L) exhibits an eccentric foam-cell rich lesion in the proximal coronary artery with adventitial inflammatory cells. This lesion is quite similar to the lesion detected in the identical artery location at 6 months (7), but with lesion progression characterized by intimal smooth muscle cells, plaque micro-thrombi and hemorrhage surrounding macrophage foam cells (Fig. 6F). In contrast to end-stage lesions in Figs. 1, 3 with marked MMP3 expression and minimal -TIMP3 expression in macrophage foam cells, macrophage MMP3 expression (Fig. 6G) is less than macrophage TIMP3 
expression (Fig. 6H). Unlike MMP3 (Fig. 6G), TIMP3 expression is not detected in smcs (Fig. 6H). A third lesion phenotype (Fig. 6I-K) also exhibits intimal $\alpha$-actin stained smcs (Fig. 6J) and macrophage foam cells at the lesion base resembling the endstage lesion phenotype with smc-rich cap (Fig. 3) but with much less lesion volume (Fig. 6I). Additionally, $\alpha$-actin stained smcs are detected in the lesion-side media, in contrast to the end-stage lesions wherein minimal smcs are detected in the lesion-side media (Fig. 3A, B). Immunohistochemical staining detects MMP3 (Fig. 6K) and VEGF (Fig. 6L) in macrophage foam cells, but no neovessel formation in contrast end-stage lesions (Fig. 3).
In all three representative lesion phenotypes in Tg53-lsd rats, disrupted internal elastic laminae (Fig. 6), neutrophil adhesion, prominent neovessel formation, and lesion rupture or erosion were not detected. Additionally, the number of apoptotic cells is reduced in non-end-stage Tg53-lsd lesions compared with end-stage Tg53 lesions. Apoptotic TUNEL $(+)$ cells are prominent in end-stage lesion (Fig. 7A-C) especially in a site of rupture. In contrast, TUNEL(+) staining is less in Tg53-lsd lesions (Fig. 7D) and lesion-side media (Fig. 7E). Other Tg53-lsd lesions are TUNEL(-) (Fig. 7F, G).

In order to quantitatively depict differential model lesion phenotypes, we used a model lesion-

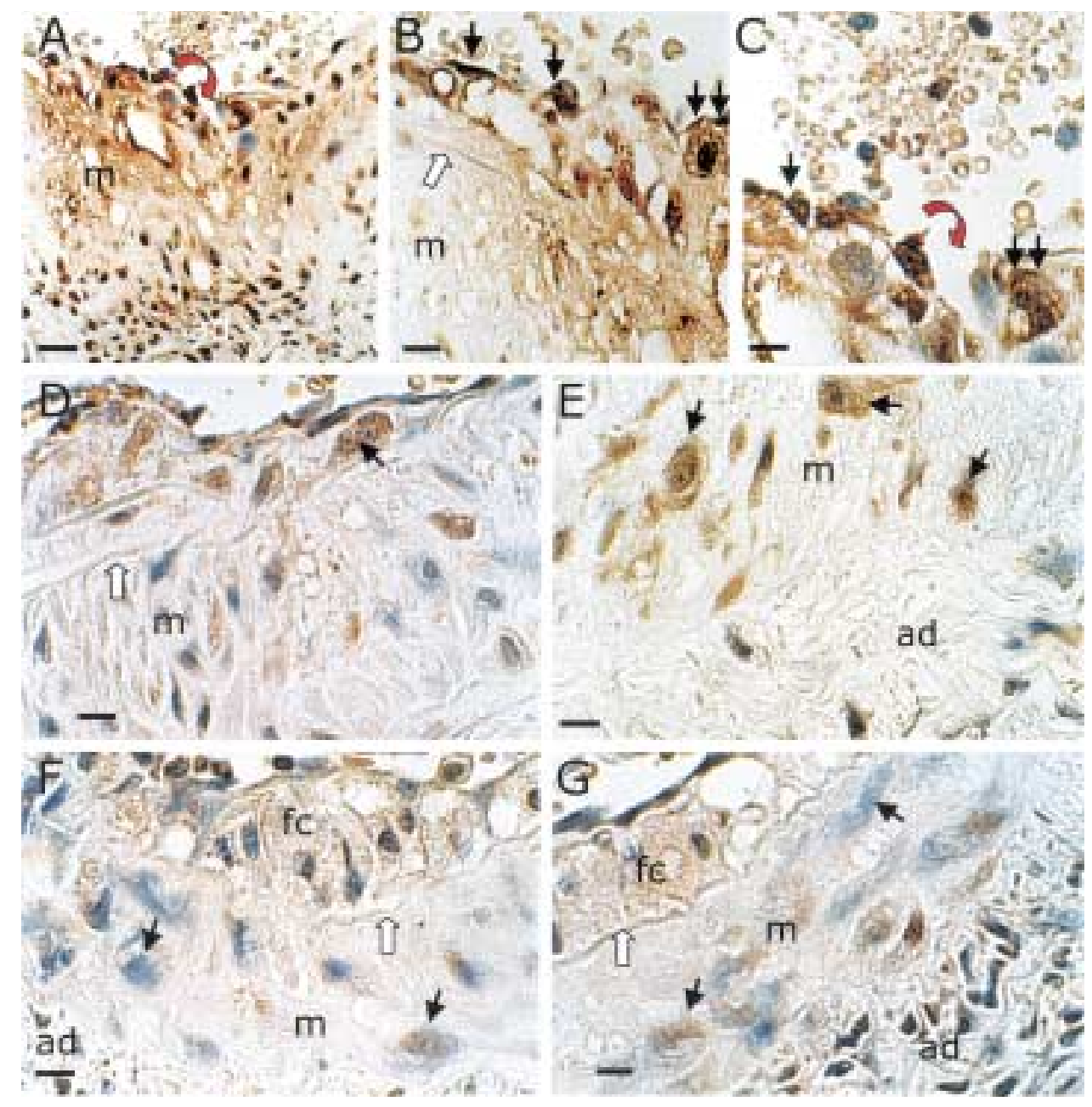

Fig. 7. Comparative analysis of TUNEL staining for apoptosis in end-stage Tg53 (A-C) vs. non-end-stage Tg53-lsd (D-G) rats. (A) End-stage Tg53 lesion exhibits TUNEL(+) (dark brown nuclei) cells close to site of rupture (red $\hookrightarrow$ ), and in extracellular lipid noted in lesion. TUNEL (-) (blue nuclei) cells are few in this region of rupture; bar $=25$ microns. (B) High magnification (bar $=10$ microns) of Panel A reveals TUNEL $(+)$ endothelial cells $(\downarrow)$ and macrophage foam cells $(\downarrow \downarrow)$ in area with endothelial erosion. (C) Another high magnification of Panel A demonstrates TUNEL $(+)$ endothelial cells $(\downarrow)$ and macrophage foam cells $(\downarrow \downarrow)$ close to disrupted lesion surface (red $\left.\subset_{>}\right)$; TUNEL $(-)$nuclei (blue); bar $=10$ microns. (D) and (E) High magnification of non-end-stage Tg53-lsd proximal coronary lesion (shown in Fig. 6, I-L) reveals mild TUNEL(+) stained cells in intimal lesion and lesion side medial smooth muscle cells $(\rightarrow)$; bars $=10$ microns. $(\mathbf{F})$ and $(\mathbf{G})$ High magnification views of Tg53-lsd lesions (shown in Fig. 6A and 6E respectively) reveal minimal, if any, TUNEL $(+)$ macrophage foam cells and smooth muscle cell $(\rightarrow)$; TUNEL $(-)$ cells exhibit blue nuclei in contrast; bars $=10$ microns. $(\Rightarrow)$, internal elastic laminae; $(\mathrm{ad})$, adventitia; $(\mathrm{fc})$, foam cells; $(\mathrm{m})$, media. 
Table 2. Phenotype component scale of coronary artery disease

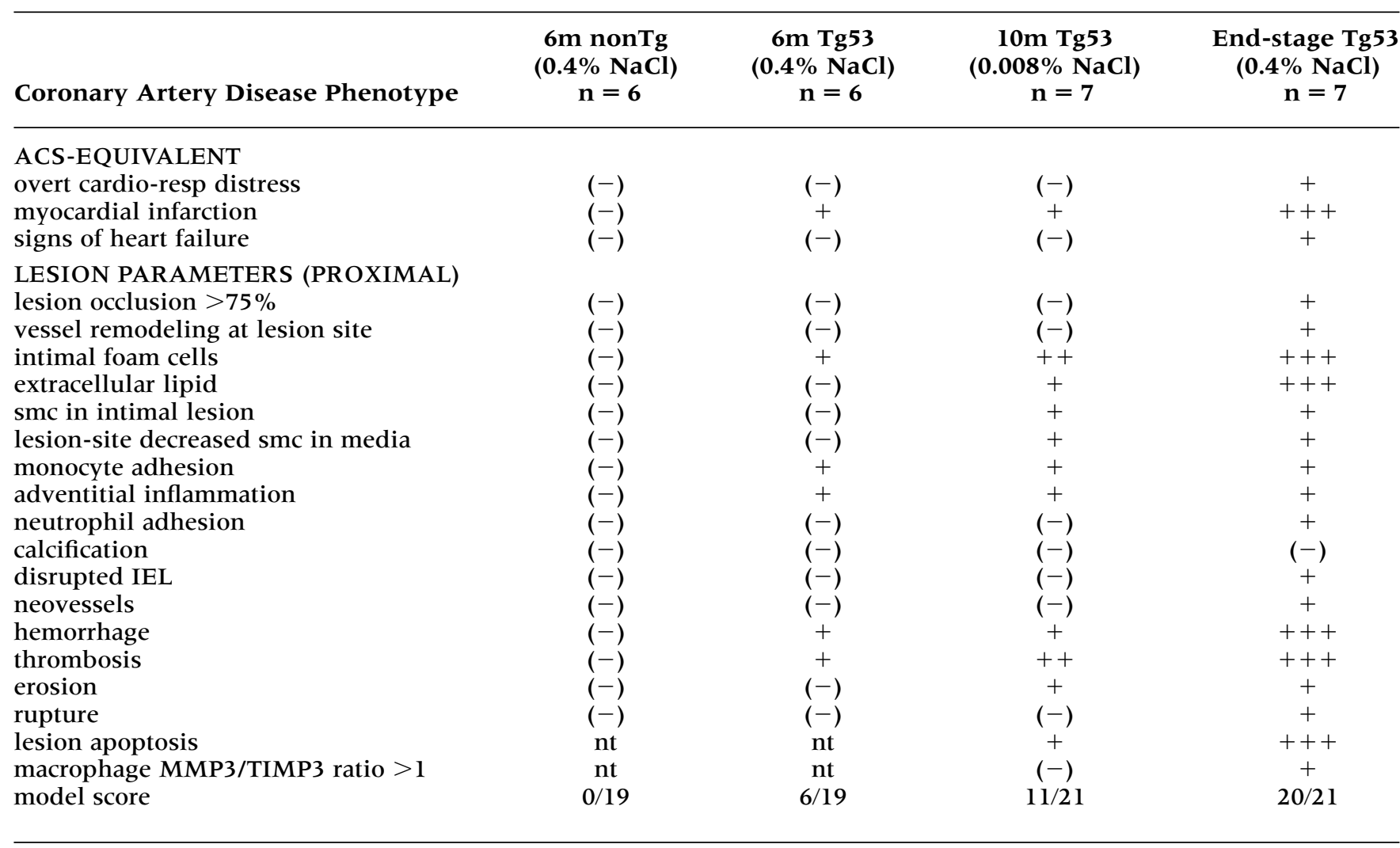

Legend: ACS, acute coronary syndromes; cardio-resp, cardio-respiratory; IEL, internal elastic lamina; MMP3, matrix metalloproteinase 3; non-tg, non-transgenic; nt, not tested; TIMP3, tissue inhibitor of metalloproteinases 3; smc, smooth muscle cells; (0.4\% NaCl), regular rat chow salt content; $(0.008 \% \mathrm{NaCl})$, low salt diet; + , present; $(-)$, not detected; relative grades used only when present in more than one study group: $+<++<+++$

spectrum scale (Table 2) depicting pertinent lesion features associated with "culprit" coronary plaques in humans with distinction of clinically overt phase of lesion progression, rather than quantifying lesion area. Because of the combinatorial permutations and lesion heterogeneity detected at end-stage simulating human acute coronary syndromes, each parameter is scored for presence or absence in the model and totaled, rather than grouped into lesion categories. When a parameter is present in more than one time point or model, a relative score is provided. Scoring proximal coronary lesions with a complete panel of serial sections obtained per heart, we compared Tg53-lsd rats with asymptomatic nonTg controls, asymptomatic $\operatorname{Tg} 53$ rats at 6 months, and end-stage Tg53 rats-the latter 3 groups on regular rat chow. Lesion spectrum scale scores revealed the following order: nonTg-reg $<6$ month-old Tg53-reg $<10$ monthold Tg53-lsd $<$ end-stage Tg53-reg rats (Table 2 ).

\section{Lipid Profile Correlates of Tg53 Lesion Phenotype}

Marked hypercholesterolemia and hypertriglyceridemia in Tg53 rats, previously reported at 6 months (7) is detected at 4 months of age in contrast to non- transgenic Dahl $\mathrm{S}$ rats $(\mathrm{P}<0.0001)$. Analysis was done at 4 months to minimize variability from decreased food intake associated with impending cardiac compromise at end-stage. Analysis of lipoprotein subclasses separated by density shows differential cholesterol distribution in the different subclasses (Table 1). The increase in cholesterol and triglyceride levels is detected predominantly in very low density lipoprotein (VLDLc and VLDLtg) fraction on regular or low salt diets. VLDLc increased from $5 \%$ in non-transgenic Dahl S rats to $84 \%$ of total plasma cholesterol in age-matched $\mathrm{Tg} 53$ rats $(\mathrm{P}<0.0001)$. VLDLtg increased from $55 \%$ to $90 \%$ of plasma triglyceride $(\mathrm{P}<0.0001)$. Both low density lipoprotein cholesterol (LDLc) and high density lipoprotein cholesterol (HDLc) are markedly decreased $(\mathrm{P}<0.0001)$ in $\mathrm{Tg} 53$ rats with a resultant increase in LDLc/HDLc ratios in Tg53 rats (LDLc/HDLc > 1), in contrast to higher LDLc and HDLc levels, but lower LDL/HDL ratio $(<1)$ in non-tg controls Dahl S rats. Negative staining transmission electron microscopy of lipoprotein particles detects larger VLDL particles and smaller LDL particles in $\mathrm{Tg} 53$ rats compared with agematched non-tg controls. HDL particle size was 
unchanged (Table 1). VLDL and HDL particle numbers increased while LDL particle number decreased compared with age-matched non-transgenic Dahl S controls. Measurement of fasting insulin/glucose ratios between $\operatorname{Tg} 53$ and non-transgenic Dahl S rats identified equivalent ratios, thus eliminating differential insulin resistance as experimental confounder (data not shown).

\section{Discussion}

\section{Insights into Coronary Lesion Development}

The detection of "culprit" plaque features which simulate observations in human acute coronary syndromes in all seven hypertensive-hyperlipidemic $\mathrm{Tg} 53$ rats at end-stage, associated with overt signs of cardio-respiratory compromise, validates the Tg53 rat model as a model of acute coronary syndromes. Said modeling implies the "capturing" of pathogenic determinants of hypertension-atherosclerosis interaction leading to coronary artery disease exacerbation.

Comparative analysis of lesion progression between proximal and distal coronary lesions at 6 months and at end-stage reveals two distinct lesion phenotypes. The first, represented by the proximal coronary lesions, are non-occlusive eccentric plaques at 6 months but progress rapidly towards "culprit" plaques at end-stage simulating complication-prone human unstable or "vulnerable" plaques $(8,10)$. The second, represented by the distal coronary lesions, are occlusive fibroproliferative lesions at 6 months which remain relatively unchanged from the 6 month time point to end-stage, simulating human stable complication-resistant plaques (5) which do not exhibit plaque hemorrhage, thrombosis, erosion or rupture, neutrophil adhesion and/or neovascularization. The contemporaneous detection at 6 months and at endstage of both lesion phenotypes (Figs. 1, 2) supports the hypothesis that complication-prone and complication-resistant plaques most likely involve distinct lesion development programs marked by different predilection sites, time course, cellular and molecular features. Intra-species vessel-specific genetic predisposition to lesion-type specific development has been observed in mice (16), rabbits (17), pigeons (18), and non-human primates (19). This notion of vesselspecific factors of lesion development recapitulates vessel-specific development (12).

\section{Hypertension Forwards Plaque Destabilization}

The effects of decreased hypertension on survival and on the coronary lesion phenotype were quite robust and admittedly unexpected. Increased survival of $\mathrm{Tg} 53$ rats fed low salt $(0.008 \% \mathrm{NaCl})$ associated with significant alteration of proximal "culprit"-prone coronary lesions and reduced hypertension suggests that polygenic (essential) hypertension can significantly accelerate coronary plaque progression. This observation is consistent with the "dose-response-like" relationship between systemic blood pressure and risk for coronary heart disease described for men age 55 years for a given level of cholesterol (1). This dose-response-like relationship is quite striking, if not counterintuitive, since both essential (polygenic) hypertension and atherosclerosis are multifactorial diseases whose interaction would be expected to be doubly complex due to the putative involvement of two sets of multifactorial determinants.

Although lesions are detected in the proximal left coronary artery system, the predominance of lesions in the non-hypertrophied right ventricle in the $\mathrm{Tg} 53$ rat model is quite intriguing. It might relate to differential features of the left and right rat coronary artery anatomy resulting in differential shear stress. This notion remains to be tested. Nevertheless, the data indicate that lesion exacerbation by hypertension detected in the right ventricle is not dependent on myocardial hypertrophy-based mechanisms since the right ventricle is not hypertrophied in systemic hypertension. The data would suggest that hypertensive vascular pathobiology provides a permissive milieu to coronary plaque progression and destabilization. Impact on lesion initiation remains to be analyzed.

Pinpointing the standout differences in proximal coronary artery lesions brought on by decrease in hypertension highlights differential plaque neutrophil adhesion, lesion apoptosis burden, macrophage foam cell MMP3/TIMP3 balance, plaque pathological neovascularization as key elements of progression to overt coronary artery disease. A parallel differential profile is also observed between end-stage proximal "culprit" and distal stable coronary artery lesions providing further supportive evidence. Since these lesion features have each been associated with "culprit" plaque progression in humans $(8-10,14,20,21)$, the association of their partial resolution with increased survival in the Tg53 rat model validates a framework for intervention targets.

\section{Lipid Profile Correlates of Severe CAD Phenotype in Tg53 Rats}

The severe coronary artery disease phenotype of Tg53 rats indicates that its lipid profile, induced by transgene overexpression of human cholesteryl ester transfer protein, represents critical proatherogenic determinants. Analyzing $\mathrm{Tg} 53$ rat lipid profiles reveals several well-established risk factors: low HDL, hypercholesterolemia, increased TC/HDL ratios, as well as several less-established risk factors: hypertriglyceridemia + low HDL, hypertriglyceridemic (large) VLDL and small LDL, and high LDL/HDL ratio albeit with low LDLc levels. The association of high LDL/HDL ratios, albeit decreased LDLc levels, and hypertriglyceridemia in $\mathrm{Tg} 53$ rats recapitulates the association of high LDL/HDL ratio and high plasma triglyceride levels with the highest risk for coronary artery disease (22). The marked elevation in VLDLc and VLDLtg in the presence of low LDLc, 
suggests that VLDL contributes significantly to the coronary heart disease phenotype in hypertensivehyperlipidemic $\operatorname{Tg} 53$ rats. This is concordant with studies demonstrating the importance of VLDL and hypertriglyceridemia in human coronary artery disease $(23,24)$, as well as the association of elevated VLDL or IDL with severity of coronary artery disease (24). This is further supported by observations in rabbit models demonstrating that increased VLDL results in higher levels of oxidized lipoproteins and a more rapid progression of aortic lesions compared with similar increase in LDL (25). The detection of large VLDL and small LDL in Tg53 rats is consistent with observations that hypertriglyceridemic large VLDL is the precursor of small LDL (26). Small LDL is linked to atherogenic process through faster influx into the arterial wall and greater susceptibility to oxidation (27). Additionally, VLDL particles rich in cholesterol, as observed in $\mathrm{Tg} 53$ rats, have a stronger atherogenic potential than VLDL rich in triglyceride (24). Altogether, concordance of Tg53 rat lipid profile with multiple proatherogenic determinants in humans further validates model-derived insights.

\section{Lesion Heterogeneity-An Indicator of Dynamic} Lesion Continuum

The spectrum of lesion features associated with "culprit" plaques detected in human acute coronary syndromes-foam cell enrichment, plaque hemorrhage and thrombosis, lesion shoulder endothelial erosion, endothelial activation and neutrophil adhesion, smooth muscle cell and macrophage expression of matrix metalloproteinase- 3 expression, imbalance of MMP3/TIMP 3 ratio, disrupted plaque endothelium and neutrophil adhesion, plaque fragile neovascularization, and rupture-associated apoptosis-indicates that a dynamic multifactorial process is in play requiring multi-faceted detection strategies, and intervention-prevention strategies. The detection of said end-stage lesion heterogeneity, in a model wherein genetic background and environmental factors are tightly controlled to be identical, suggests either a missed unregulated factor or a putative stochastic paradigm in plaque progression. A stochastic paradigm would provide a framework to observations of seemingly disparate "culprit" plaque hallmarks in humans $(4,8,10)$. Nevertheless, recapitulating lesion heterogeneity, as observed in humans $(4,28)$, in a regulated experimental model translates into an imperative need for trans-lesion analysis that can detect ongoing lesion destabilization in multiple sites and at various stages. Likewise, the notion of singleton marker or singleton/best therapy becomes a greater challenge given the emerging dynamic continuum of pathogenic mechanisms and events depicted in genetically identical and environmentally controlled $\mathrm{Tg} 53$ rat model at end-stage.

These model-derived insights provide compelling experimental evidence in support of parallel perspectives on human acute coronary syndromes
$(8,10)$. Altogether, the data unite previous observations into a clinically relevant hierarchical hypothesis that given equivalent hyperlipidemia and genetic susceptibility, polygenic hypertension forwards coronary artery disease progression, complication and destabilization. These paradigmatic insights are critical especially given the complexities of coronary death (4) and the unequivocal clinical importance of understanding said pathobiology (8).

\section{Acknowledgments}

This work was supported by USPHS grant HL62857 and NIH HL 58833.

\section{References}

1. MRFIT: Multiple Risk Factor Intervention Trial Research Group. Relationship between baseline risk factors and coronary heart disease and total mortality in the Multiple Risk Factor Intervention Trail. (1986) Prev. Med. 15: 254-273.

2. Casteli WP, Anderson KA. (1986) A population at risk: prevalence of high cholesterol levels in hypertensive patients in the Framingham Study. Am. J. Med. 80(Suppl 2A): 23-32.

3. Getz GS. (2000) Mouse model of unstable atherosclerotic plaque? Arterioscler. Thromb. Vasc. Biol. 20: 2503-2505.

4. Virmani R, Koldogie FD, Burke AP, et al. (2000) Lessons from sudden coronary death: A comprehensive morphological classification scheme for atherosclerotic lesions. Arterioscler Thromb. Vasc. Biol. 20: 1262-1275.

5. Nickerson CJ, Haudenschild CC, Chobanian AV. (1992) Effects of hypertension and hyperlipidemia on the myocardium and coronary vasculature of the WHHL rabbit. Exp. Mol. Pathol. 56: $173-185$.

6. Knowles JW, Reddick RL, Jennette JC, et al. (2000) Enhanced atherosclerosis and kidney dysfunction in $\mathrm{eNOS}^{-1-}$, ApoE $^{-1-}$ mice are ameliorated by enalapril treatment. J. Clin. Invest. 105: 451-458.

7. Herrera VLM, Makrides SC, Xie HX, et al. (1999) Spontaneous combined hyperlipidemia, coronary heart disease and decreased survival in Dahl salt-sensitive hypertensive rats transgenic for human cholesteryl ester transfer protein. Nature Med. 5: 383-1389.

8. Libby P, Geng YJ, Sukhova GK, et al. (1997) Molecular determinants of atherosclerotic plaque vulnerability. Ann. N.Y Acad. Sci. 811: 134-142.

9. Kolodgie FD, Narula J, Burke AP, et al. (2000) Localization of apoptotic macrophages at the site of plaque rupture in sudden coronary death. Am. J. Pathol. 157: 1259-1268.

10. Delager-Pedersen S, Pederson EM, Ringgaard S, Falk E. (1999) Coronary artery disease: plaque vulnerability, disruption and thrombosis. In: The Vulnerable Atherosclerotic Plaque, ed. V. Fuster, Futura Publishing Co., Inc., New York, NY, pp. 1-23.

11. Henney AM, Wakeley PR, Davies MJ, et al. (1991) Localization of stromelysin gene expression in atherosclerotic plaques by in situ hybridization. Proc. Natl. Acad. Sci. USA 88: 8154-8158.

12. Yancopoulos GD, Davis S, Gale NW, et al. (2000) Vascularspecific growth factors and blood vessel formation. Nature 407: $242-248$.

13. deBoer OJ, van der Wal AC, Teeling P, Becker AE. (1999) Leukocyte recruitment in rupture prone regions of lipid-rich plaques: a prominent role for neovascularization? Cardiovasc. Res. 41: 443-339.

14. Inoue M, Itoh H, Ueda M, et al. (1998) Vascular endothelial growth factor (VEGF) expression in human coronary atherosclerotic lesions: possible pathophysiological significance of VEGF in progression of atherosclerosis. Circulation 98: 2108-2116. 
15. Overbeck HW, Ku DD, Rapp JP. (1981) Sodium pump activity in arteries of Dahl salt-sensitive rats. Hypertension 3: 306-312.

16. Qiao J-H, Xie P-Z, Fishbein MC, et al. (1994) Pathology of atheromatous lesions in inbred and genetically engineered mice. Arterioscler. Thromb. 14: 1480-1497.

17. Shiomi M, Ito T, Shiraishi M, Watanabe Y. (1992) Inheritability of atherosclerosis and the role of lipoproteins as risk factors related to coronary atherosclerosis are different from those related to aortic atherosclerosis. Atherosclerosis 96: 43-52.

18. Wagner WD, Clarkson TB, Feldner MA, Prichard RW. (1973) The development of pigeon strains with selected atherosclerosis characteristics. Exper. Mol. Path. 19: 304-319.

19. Bullock BC, Lehner NDM, Clarkson TB, et al. (1975) Comparative primate atherosclerosis. Exper. Mol. Path. 22: 15 1-175.

20. Kockx MM, Herman AG. (1998) Apoptosis in atherogenesis: implications for plaque destabilization. Eur. Heart J. (Suppl G): G23-G28.

21. George SJ. (1998) Tissue inhibitors of metalloproteinases and metalloproteinases in atherosclerosis. Curr. Opinion in Lipidology 9: 413-423.
22. Ooi TC, Ooi DS. (1998) The atherogenic significance of an elevated plasma triglyceride level. Crit. Rev. Clin. Lab. Sci. 35(6): 489-516.

23. Krauss RM. (1998) Triglycerides and atherogenic lipoproteins: Rationale for lipid management. Am. J. Med. 105: 58S-62S.

24. Chanu B. (1999) Hypertriglyceridemia: danger for the arteries. Presse. Med. 28: 2011-2017.

25. Holvoet P, Collen D. (1997) beta-VLDL hypercholesterolemia relative to LDL hypercholesterolemia is associated with higher levels of oxidized lipoproteins and a more rapid progression of coronary atherosclerosis in rabbits. Arterioscler. Thromb. Vasc. Biol. 17: 2376-2382.

26. Demant T, Packard C. (1998) In vivo studies of VLDL metabolism and LDL heterogeneity. Eur. Heart J. 19(Suppl H): H7-H10.

27. Griffin BA. (1999) Lipoprotein atherogenicity: An overview of current mechanisms. Proc. Nutr. Soc. 58:163-169.

28. Goldstein JA, Demetriou D, Grines CL. (2000) Multiple complex coronary plaques in patients with acute myocardial infarction. New Engl. J. Med. 343: 915-922. 